BULLETIN Bulletin hispanique

HispaniQuE Université Michel de Montaigne Bordeaux

116-1 | 2014

Varia

\title{
Les musées d'histoire naturelle et la construction des identités nationales en Amérique Latine
}

l'influence des naturalistes européens en Argentine, au Chili et en Uruguay au moment des Indépendances

\section{Cécile Petit}

\section{CpenEdition}

\section{Journals}

Édition électronique

URL : http://journals.openedition.org/bulletinhispanique/3199

DOI : 10.4000/bulletinhispanique.3199

ISSN : $1775-3821$

Éditeur

Presses universitaires de Bordeaux

\section{Édition imprimée}

Date de publication : 1 juin 2014

Pagination : 319-337

ISBN : 978-2-86781-931-5

ISSN : 0007-4640

Référence électronique

Cécile Petit, «Les musées d'histoire naturelle et la construction des identités nationales en Amérique Latine », Bulletin hispanique [En ligne], 116-1 | 2014, mis en ligne le 01 juin 2017, consulté le 26 mai 2020. URL : http://journals.openedition.org/bulletinhispanique/3199 ; DOI : https://doi.org/10.4000/ bulletinhispanique.3199 


\title{
Les musées d'histoire naturelle et la construction des identités nationales en Amérique Latine l'influence des naturalistes européens en Argentine, au Chili et en Uruguay au moment des Indépendances
}

\author{
Cécile Petit \\ Criccal Université Paris III - Université d'Éry
}

Pendant les premières décennies qui suivent les Indépendances latino-américaines, la fondation de musées d'histoire naturelle a pour objectif de créer un patrimoine national, de l'institutionnaliser, afin de contribuer à la construction identitaire de la nation. La création de ces musées s'appuie sur l'expérience de naturalistes européens ou formés en Europe.

Mots-clés : histoire naturelle, science, musée, identité nationale, migration des élites, Amérique Latine.

Durante las primeras décadas que siguieron las Independencias latinoamericanas, se fundaron museos de historia natural con el objetivo de crear un patrimonio nacional, de institucionalizarlo, para que contribuyeran a la construcción identitaria de la nación. La creación de estos museos se apoyó en los aportes de naturalistas europeos o formados en Europa.

Palabras claves: historia natural, ciencia, museo, identidad nacional, migración de las élites, América Latina.

In the first decades following the Independence of Latin American countries, natural history museums were founded to create a national heritage, institutionalize it, and contribute to the construction of national identities. The creation of these museums rested on the contributions of European naturalists or of naturalists trained in Europe.

Keywords: natural history, science, museum, national identity, migration of elites, Latin America. 
$\mathrm{A}_{\text {par différents moyens de se forger une identité propre afin de prendre }}^{\text {u moment de leur Indépendance, les républiques latino-américaines tentent }}$ leurs distances vis à vis de l'Espagne et de se démarquer les unes des autres. La création de musées, et tout particulièrement de musées d'histoire naturelle, fait partie des stratégies mises en oeuvre pour mener à bien cette construction identitaire, les muséums ayant pour rôle d'affirmer et d'institutionnaliser l'existence d'un patrimoine national. La démarche non seulement scientifique mais aussi et surtout politique entreprise par les pays latino-américains nouvellement indépendants, va s'appuyer sur l'expérience de naturalistes européens ou formés en Europe. Afin de montrer l'importance et l'impact de la migration des naturalistes européens en Amérique Latine pendant les premières décennies suivant les Indépendances, afin de comprendre dans quelle mesure leur présence a favorisé l'émancipation scientifique et identitaire des pays latino-américains ou a renforcé leur dépendance vis à vis de l'Europe, nous nous intéresserons à la fondation des muséums du Cono Sur, à savoir le Museo Argentino de Ciencias Naturales Bernardino Rivadavia, le Museo Nacional de Historia Natural du Chili, le Museo Nacional de Historia Natural y Antropología d'Uruguay. Nous concentrerons notre attention sur le contexte de ces créations et le rôle identitaire de ces musées, sur l'origine et la formation des premiers naturalistes y ayant pris part, puis sur leurs apports et leurs dépendances.

\section{LES MUSÉES D'HISTOIRE NATURELLE ET LA CONSTRUCTION DES IDENTITÉS NATIONALES AU MOMENT DES INDÉPENDANCES}

Lors des Indépendances, les nouvelles républiques latino-américaines s'efforcent de légitimer leur création et de justifier les frontières qui les séparent grâce à la construction d'une identité nationale. La constitution de cette identité, nécessaire à la formation de la nation, se fait par le biais d'une politique culturelle $^{1}$ qui comprend notamment la connaissance et la reconnaissance des ressources du territoire, l'exploration et l'institutionnalisation du patrimoine. La création des musées d'histoire naturelle est directement issue de cette politique culturelle visant à construire l'identité nationale, car le muséum est une institution permettant de rassembler, protéger, préserver, conserver, étudier, exposer, valoriser la richesse et les éléments naturels représentatifs de l'identité naturelle et biologique d'un pays. Récolter, examiner, classifier, présenter le maximum d'espèces propres à un territoire sont des pratiques qui participent à l'élaboration et à l'exaltation d'une identité considérée comme unique. En mettant en avant l'existence d'une nature qui lui est propre, en la

1. Ce qu'écrit Mizón au sujet du Chili peut également s'appliquer à l'Argentine et à l'Uruguay: «La formación del Estado republicano coincide con la formación de la política cultural chilena ; ambos fenómenos son inseparables, forman parte del mismo proyecto ». Luis Mizón, Claudio Gay y la formación de la identidad cultural chilena, Santiago de Chile, Editorial Universitaria, 2001, 175 p., p. 28. 
présentant officiellement comme le patrimoine de toute une communauté, le musée d'histoire naturelle a pour mission de réunir la population autour d'une identité qui serait commune, spécifique, et de revendiquer sa différence par rapport à l'ancienne métropole et aux pays voisins. La formation des premiers muséums latino-américains se produit ainsi au moment des Indépendances ou juste après. C'est en particulier le cas des trois pays sur lesquels s'est portée notre attention : l'Argentine, le Chili et l'Uruguay.

L'indépendance de l'Argentine a lieu en 1810, elle est officialisée en 1816, mais c'est dès 1812 qu'apparaît le projet de création d'un musée d'histoire naturelle. Ce muséum, aujourd'hui nommé Museo Argentino de Ciencias Naturales Bernardino Rivadavia, s'appelle à l'époque Museo de Ciencias Naturales. Le nom de Bernardino Rivadavia sera ajouté en 1923, ce qui souligne le rapport existant entre la création du muséum et la naissance de la nation puisque Rivadavia, premier président de la République Argentine (18261827) et secrétaire à la guerre du Premier Tiumvirat (1811), est à l'origine de la fondation du musée de sciences naturelles. Sous l'impulsion de Rivadavia, le Premier Triumvirat émet en 1812 une circulaire incitant les gouverneurs des Provinces Unies du Río de la Plata à contribuer à la création du Muséum de la capitale puis, en décembre 1823, Rivadavia, alors ministre de Martín Rodríguez, signe une résolution visant à adopter des mesures afin d'accélérer la création du Musée ${ }^{2}$. L'indépendance du Chili est quant à elle proclamée en 1810 avant d'être entérinée en 1818. Le Museo Nacional de Historia Natural est créé quelques années après, en 1830, à la demande du gouvernement chilien qui souhaite procéder à une étude exhaustive des ressources du pays et fonder un muséum où les exposer afin que la connaissance et l'institutionnalisation des richesses végétales et minérales participent à la construction de l'identité nationale ${ }^{3}$. Pour ce qui est de l'Uruguay, il revendique son indépendance en

2. Voir : Museo Argentino de Ciencias Naturales Bernardino Rivadavia, La historia del museo. In Museo Argentino de Ciencias Naturales Bernardino Rivadavia. Site du Museo Argentino de Ciencias Naturales. [En ligne]. [Consulté le 22 février 2013]. Disponible sur : http://www.macn. secyt.gov.ar/elmuseo/historia.php

3. Ainsi, on peut lire l'indication suivante sur le site de la Biblioteca del Congreso Nacional de Chile : "Junto con las preocupaciones urgentes que el proceso de independencia implicó para el país y que decían relación con la consolidación militar de la emancipación y la búsqueda de una institucionalidad que diera cuenta de la transición hacia formas de vida republicana, las autoridades gubernamentales de aquella época advirtieron la necesidad de conocer y explorar los vastos territorios de Chile como una forma de impulsar una cierta identidad colectiva que sustentara la consolidación de una nación y posteriormente de un Estado. " Biblioteca del Congreso Nacional de Chile. Claudio Gay y su obra 'Historia Física y Política de Chile'. Site de la Biblioteca del Congreso Nacional de Chile. [En ligne]. [Consulté le 22 février 2013]. Disponible sur : http://www.bcn.cl/bibliodigital/dhisto/cgay

Voir également le site suivant : Dirección de Bibliotecas, Archivos y Museos. Museo Nacional de Historia Natural de Chile. Historia. In Dirección de Bibliotecas, Archivos y Museos. Museo Nacional de Historia Natural de Chile. Site du Museo Nacional de Historia Natural. [En ligne]. [Consulté le 22 février 2013]. Disponible sur : http://www.mnhn.cl/Vistas_Publicas/ publicContenido/contenidoPublicDetalle.aspx?folio=3906\&idioma=0. J'ai aussi consulté l'étude de Rosario Mena: Rosario Mena. La cruzada de un viajero. Claudio Gay y su Historia 
1811 mais, faisant tantôt partie des Provinces Unies du Río de la Plata tantôt du Brésil, c'est en 1828 qu'il devient un État indépendant. Par décret, une commission est mise en place en 1837 en vue de réaliser un Musée National d'Histoire Naturelle ${ }^{4}$. La création de ces muséums intervient donc au moment des Indépendances ou au cours des années qui suivent pour répondre au besoin de ces nouvelles républiques de se construire comme nation autour d'une identité spécifique. Elle reposera cependant sur le travail de naturalistes européens ou formés en Europe.

\section{LES FONDATEURS DES MUSÉES D'HISTOIRE NATURELLE : ORIGINE ET FORMATION DES NATURALISTES}

La fondation de ces muséums s'appuie en effet sur des scientifiques ayant étudié en Europe ou venus d'Europe pour les créer et les diriger, procéder à des expéditions, inventorier et étudier les spécimens recueillis. Les naturalistes qui participent aux débuts de ces musées sont soit des Latino-américains issus de l'élite criolla ayant reçu une éducation européenne et s'étant en partie formés en Europe, soit des Européens œuvrant à titre personnel ou recrutés par les gouvernements chilien, argentin ou uruguayen ${ }^{5}$ en raison de la difficulté à trouver des scientifiques latino-américains en mesure de réaliser ce type de projet ou s'y intéressant ${ }^{6}$. Ainsi, le Museo Argentino de Ciencias Naturales a pour directeur initial un italien, Carlos Ferraris (1793-1859), également appelé Pablo Ferrari ou Cadmio Ferraris selon les sources, qui gèrera le musée de

Física y Política de Chile. In Corporación Patrimonio Cultural de Chile. Nuestro.cl. Juin 2004. [En ligne]. [Consulté le 22 février 2013]. Disponible sur : http://www.nuestro.cl/notas/rescate/ claudio_gay $1 . h$ tm

4. Á ce sujet, voir le travail de Álvaro Mones. Reseña histórica del Museo Nacional de Historia Natural. In Museo Nacional de Historia Natural y Antropología de Uruguay. Site du Museo Nacional de Historia Natural y Antropología de Uruguay. [En ligne]. [Consulté le 22 février 2013]. Disponible sur : http://www.mec.gub.uy/munhina/historia.htm

5. J'ai consulté le livre de Mauricio Nieto, Remedios para el imperio: historia natural y la apropiación del Nuevo Mundo, Bogotá, ICANH, 2000, 280 p., p. 242-243, 257, ainsi que l'ouvrage de Sol Serrano, Universidad y nación: Chile en el siglo XIX, Santiago de Chile, Editorial Universitaria, 1994, 276 p., p. 113. Voir également le site de la Biblioteca del Congreso Nacional de Chile, loc. cit., l'article de Rosario Mena, loc. cit., et le site suivant: Facultad de Matemática, Astronomía y Física. Universidad Nacional de Córdoba. Carta Molina, Pedro. In Facultad de Matemática, Astronomía y Física. Programa de divulgación científica. [En ligne]. [Consulté le 22 février 2013]. Disponible sur: http ://divulgacion.famaf.unc.edu.ar/?q=ameghino/cartamolina-pedro

6. Rafael Sagredo Baeza et Juan María Gutiérrez se réferrent à cet aspect. Voir : Rafael Sagredo Baeza, «El arte de un naturalista », El Mercurio, 4 juillet 2004. In El Mercurio. Site de El Mercurio. [En ligne]. [Consulté le 22 février 2013]. Disponible sur http://diario.elmercurio. $\mathrm{cl} /$ detalle/index.asp?id=\{2a84fe84-98a7-475e-9390-beea7c75c6cd $\}.$ Voir également: Juan María Gutiérrez, "El Dr. D. Teodoro M. Vilardebó ", Apuntes biográficos de escritores, oradores y hombres de estado de la República Argentina, Argentina, Imprenta de Mayo, 1860, 297 p., p. 275-284, p. 276. 
1826 à 1842. Le naturaliste français Alcide d'Orbigny (1802-1857) participera aux premiers travaux du muséum ${ }^{7}$. Le Museo Nacional de Historia Natural du Chili est quant à lui créé et dirigé par un français, Claude Gay (1800-1873), embauché par le gouvernement chilien en $1830^{8}$; il en assurera la direction jusqu'en 1842. Teodoro M. Vilardebó (1803-1857), uruguayen formé en Espagne puis en France, sera le premier directeur du Musée National d'Histoire Naturelle d'Uruguay, de 1839 à 1844. Le naturaliste français Arsène Isabelle (1807-1888) participera à la première expédition organisée par le Museo Nacional de Historia Natural uruguayen'. Tous ces naturalistes reçoivent une formation qui ne sera pas sans incidence sur la façon dont ils mèneront leur travail en Amérique Latine.

Ainsi, Carlos Ferraris fait ses études à l'Université de Turin, où il obtient en 1817 le diplôme de pharmacien. Il poursuit sa formation dans différentes universités puis, pour des raisons politiques, émigre en Espagne, en France et en Belgique. Ses études lui permettent de faire la connaissance de Pedro Carta Molina, médecin et physicien italien qui l'influencera et lui proposera de se rendre à Buenos Aires pour prendre la direction d'un cabinet de sciences physiques et de sciences naturelles. Pedro Carta Molina étudie également à l'Université de Turin, il y décroche le diplôme de médecin et il y enseignera. En raison du contexte politique, il part en Espagne, en France, en Suisse, en Allemagne, en Angleterre, pays dans lesquels il se tient informé des progrès en médecine et en sciences naturelles. Alors qu'il rencontre Rivadavia en Angleterre, celui-ci, chargé de recruter des scientifiques européens pour l'Argentine, lui propose la direction du Cabinet de Physique, mais jugeant ses connaissances insuffisantes Carta Molina décide de se former davantage en Europe avant de prendre ses fonctions en Argentine. De 1824 à 1826 il étudie dans différentes universités comme celles de Heidelberg, Halle, Berlin, Göttingen, et assiste aux cours de Gay-Lussac et d'Ampère. La formation de Carlos Ferraris sera marquée par celle de Carta Molina, son mentor ${ }^{10}$.

Quant à Claude Gay, il est issu d'une famille d'agriculteurs et s'intéresse dès son enfance aux sciences naturelles. Étudier la nature deviendra pour lui une passion ${ }^{11}$. Ses parents attachant de l'importance à l'éducation et ayant les moyens

7. À ce sujet, j’ai consulté : Museo Argentino de Ciencias Naturales Bernardino Rivadavia, loc. cit.

8. Voir le site suivant : Dirección de Bibliotecas, Archivos y Museos. Museo Nacional de Historia Natural de Chile, loc. cit.

9. Voir l'étude de Álvaro Mones, loc. cit

10. J'ai consulté les pages suivantes du site de la Facultad de Matemática, Astronomía y Física: Facultad de Matemática, Astronomía y Física. Universidad Nacional de Córdoba. Ferraris, Carlos. In Facultad de Matemática, Astronomía y Física. Programa de divulgación científica. [En ligne]. [Consulté le 22 février 2013]. Disponible sur : http://divulgacion.famaf.unc.edu. ar/?q=ameghino/ferraris-carlos et Facultad de Matemática, Astronomía y Física. Universidad Nacional de Córdoba. Carta Molina, Pedro, loc. cit.

11. Claude Gay s'exprime en ces termes : «El apego con que, desde mi tierna infancia, me entregué al estudio de las ciencias, vino á ser en breve una irresistible pasion por todo cuanto en la naturaleza es digno esencialmente de un atento examen y de un análisis especial »Claudio 
de l'envoyer à l'école, il est scolarisé puis fait des études secondaires classiques. C'est un élève sérieux. À dix-huit ans, il part à Paris pour y étudier la médecine et la pharmacie mais, préférant la botanique et l'entomologie, il abandonne ses études pour se consacrer aux sciences naturelles. Ainsi, pendant huit ans, de 1821 à 1828, il suit assidûment des cours de botanique, d'entomologie mais aussi de physique, de chimie, de géologie et d'anatomie comparée au Musée d'Histoire Naturelle de Paris et à la Sorbonne. Il a notamment pour professeurs Cuvier, Desfontaines et Jussieu, qui l'encouragent à cultiver sa passion. Il travaille sous la direction du botaniste italien Giovanni Battista Balbis, professeur et directeur du Jardin des Plantes de Lyon puis devient collecteur pour le Musée d'Histoire Naturelle de Paris ${ }^{12}$.

Teodoro Vilardebó, né en Uruguay, part au Brésil avec sa famille lorsqu'il a douze ans, en 1815, au moment de la guerre d'indépendance, puis est envoyé chez ses grands-parents en Espagne, à Barcelone, pour faire ses études secondaires. Il étudie ensuite les mathématiques et la chirurgie à l'Université de Cervera. Il s'installe en France en 1825, à Paris, pour suivre des études de médecine à la Sorbonne où il obtient son Doctorat en médecine en 1830 puis en chirurgie en 1831. Il fera partie d'une commission de chercheurs français chargés de réaliser une investigation sur le choléra en Europe du Nord. Il rentre en Uruguay en 1833, sans avoir terminé son Doctorat en sciences et en pharmacie. Pendant ses années passées en France, il se forme, côtoie des professeurs de renom, se nourrit de lectures scientifiques européennes. Par la suite, en 1847, il effectuera un autre séjour en Europe, à Barcelone puis à Paris, afin d'améliorer les connaissances dont il a besoin pour mener à bien ses fonctions scientifiques en Uruguay ${ }^{13}$.

Gay, Historia Física y Politica de Chile, Historia, 21 Tomes, Paris, 1844-1871, Tomo 1, 1844, 536 p., Prólogo, p. V.

12. J'ai consulté : Claudio Gay, op. cit., p. V; Diego Barros Arana, Don Claudio Gay; su vida i sus obras, Santiago de Chile, Imp. Cervantes, Estudios Histórico-Bibliográficos, 1911, 461 p., p. 240-457, p. 272-273 ; Dirección de Bibliotecas, Archivos y Museos. Memoria Chilena. Claudio Gay (1800-1873). [En ligne]. [Consultéle 22 février 2013]. Disponible sur: http://www. memoriachilena.cl/temas/index.asp?id_ut=claudiogay(1800-1873)pionerodelacienciaenchile ; Biblioteca del Congreso Nacional de Chile, loc. cit, Rafael Sagredo Baeza, "El arte de un naturalista ", loc. cit.

13. À ce sujet, j’ai consulté les travaux suivants : Alberto F. Onna, « Estrategias de visualización y legitimación de los primeros paleontólogos en el Río de la Plata durante la primera mitad del siglo XIX: Francisco Javier Muñiz y Teodoro Miguel Vilardebó ", in La ciencia en la Argentina entre siglos: textos, contextos e instituciones, Marcelo Montserrat (compilador) Buenos Aires, Ediciones Manantial, 2000, 369 p., p. 53-68, p. 64, 66-67 ; Juan María Gutiérrez, op. cit., p. 276-277, 280-281; Alvaro Kröger. Teodoro Miguel Simón Vilardebó, recopilación sobre un texto de José María Fernández Saldaña. In: Informe Uruguay, año III, no 146, 2 septembre 2005. [En ligne]. [Consulté le 22 février 2013]. Disponible sur : http://www.uruguayinforme.com/ news/02092005/02092005kroger_historias.htm ; María L. Coolighan Sanguinetti, «Teodoro M. Vilardebó (1803-1857) ", in Médicos Uruguayos Ejemplares, Tomo II, 1989, p. 27-38, p. 27 31, 37 ; Guillermo Whittembury, Máximo Drets, Fernando Mañé-Garzón, "The only extant manuscript of Claude Bernard's first course on experimental physiology in collège de france during the winter of 1847-1848", Interciencia, marzo 2009, vol. 34, no 3, p. 219-224, p. 219. 
Arsène Isabelle est quant à lui curieux de nature ; la littérature fantastique, historique puis scientifique lui donnera le goût du voyage ${ }^{14}$. Ses lectures lui permettent aussi d'acquérir une culture générale assez vaste et d'approfondir ses connaissances en science et en histoire naturelle. Il lit notamment Rousseau, Pascal, Bernardin de Saint Pierre, Pline, Buffon, Alexander Von Humboldt, Saint Hilaire, d'Orbigny, mais aussi l'écrivain moraliste Samuel Johnson, l'historien voyageur Pierre François Xavier de Charlevoix, l'écrivain anglais Robert Southey, le naturaliste espagnol Félix de Azara, le minéralogiste voyageur John Mawe, entre autres ${ }^{15}$.

Alcide d'Orbigny passe son enfance dans un contexte scientifique car son père, Charles-Marie D’Orbigny, est médecin militaire puis médecin civil et surtout naturaliste : il consacre son temps libre à sa passion pour les sciences naturelles. Lecteur de Linné et de Lamarck, il répertorie la faune et la flore des côtes de la région de Nantes, Noirmoutiers et La Rochelle. Il crée l'Académie Ambulante de Noirmoutiers avec, en particulier, l'un des membres de la Société Académique de Loire inférieure, société qui sera à l'origine du Muséum de Nantes, et fréquente l'Académie des Belles Lettres, Sciences et Arts de La Rochelle à laquelle participent notamment le physicien Réaumur et Aimé de Bonpland, proche des d'Orbigny. En contact avec des professeurs du Muséum de Paris comme Cuvier ou Alexandre Brongniart, il correspond avec eux et leur envoie les herbiers composés par ses enfants, des inventaires de poissons, de reptiles, de crabes et des spécimens conservés dans de l'alcool. Il sera plus tard l'un des fondateurs du Musée d'Histoire Naturelle de La Rochelle et deviendra correspondant du Muséum de Paris ${ }^{16}$. Son fils, Alcide d'Orbigny, baigne ainsi depuis sa plus tendre enfance dans une atmosphère scientifique. Son père l'initiera très tôt à l'observation de la nature ${ }^{17}$ puis ils travailleront ensemble sur les coquillages microscopiques présents dans les sables, auxquels ils donneront le nom de foraminifères, et dont Alcide réalisera ensuite les croquis ${ }^{18}$. Il suit des

14. Arsène Isabelle écrit ceci : «J'ai toujours eu un penchant irrésistible pour les voyages, aussi j'en ai dévoré un grand nombre, à commencer par les Gulliver's travels jusqu'au Voyage pittoresque autour du Monde. Ces lectures ne pouvaient manquer de faire naître en moi le désir de voyager : notez en outre que je suis curieux à l'excès!.... (...) Donc je suis né curieux et cette curiosité m'a porté à voyager. " Arsène Isabelle, Voyage à Buénos-Ayres et à Porto-Alègre, par la Banda-Oriental, les missions d'Uruguay et la Province de Rio-Grande-Do-Sul, Le Havre, Impr. Jean Morlent, 1835, 618 p., p. 3-5.

15. À ce sujet voir Arsène Isabelle, op cit., p. 5-7, 22, 28 et Horacio Aguilar, "Arsenio Isabelle y su crónica sobre el museo ", El Carnotaurus, Boletín del Museo Argentino de Ciencias Naturales Bernardino Rivadavia, noviembre de 2009, año X, n 107, 16 p., p. 6-7.

16. J'ai consulté le livre de Françoise Legré-Zaidline, Alcide Dessalines d'Orbigny (18021857), Paris, L'Harmattan 2002, 249 p., p. 9, 11-17, 26, 38, 140.

17. Alcide d'Orbigny écrit ceci à son sujet : "Né avec des dispositions toutes particulières pour les sciences naturelles, dispositions qui se manifestèrent en moi dès mes premières années, je dus, aux encouragements d'un père honorablement connu du monde savant, et à ses doctes leçons, le développement prématuré de l'irrésistible instinct qui m’entraînait vers leur étude. » Alcide d'Orbigny, Voyage en Amérique méridionale, Paris/Strasbourg, P. Bertrand/Veuve Levrault, 9 Tomes, Tome 1, 1835, p. 2.

18. Voir l'étude de Françoise Legré-Zaidline, op. cit., p. 9, 27. 
études classiques à La Rochelle, ville disposant d'un jardin botanique et dont l'ambiance propice au voyage et aux expéditions naturalistes ne manquera pas de l'influencer ${ }^{19}$. En 1824, il part pour Paris et assiste aux cours de Cuvier, Saint Hilaire, de Blainville, Cordier et Brongniart ${ }^{20}$. La renommée de son père lui ouvrira des portes ${ }^{21}$. Ainsi, par le biais du baron de Férussac, membre de l'Académie des Belles Lettres, Sciences et Arts de La Rochelle et correspondant au Musée d'Histoire Naturelle de Paris, Alcide d'Orbigny obtient un poste d'aide naturaliste au Muséum de Paris en février 1825. Son travail de classification des foraminiferres présenté à l'Académie des Sciences le 7 novembre 1825 et ses solides connaissances en Histoire Naturelle attirent l'attention des savants du Muséum de Paris qui lui proposent le 16 novembre 1825 de réaliser une mission d'exploration en Amérique du Sud. Pour accomplir cette mission, il recevra une formation supplémentaire qu'il effectuera à l'École des Naturalistes Voyageurs fondée en 1818 par Thouin. Ainsi, pendant plus d'un an, il fréquentera cette école et approfondira auprès d'éminents professeurs ses connaissances en zoologie, entomologie, minéralogie, botanique, géologie, paléontologie, anatomie ${ }^{22}$.

Une fois en Amérique, ces naturalistes mettront en application les méthodes et les théories qu'ils auront apprises tout au long de leur formation, ils

19. J'ai consulté le site suivant : Musée National d'Histoire Naturelle de Paris. Alcide d'Orbigny voyageur naturaliste 1802-1857, du Nouveau Monde...au passé du monde. Exposition Alcide d'Orbigny, 2002. In: Musée National d'Histoire Naturelle de Paris. Site du Musée National d'Histoire Naturelle. [En ligne]. [Consulté le 22 février 2013]. Disponible sur : http://www.mnhn.fr/expo/orbigny/ Le Jardin des plantes de La Rochelle est créé au XVII ${ }^{e}$ siècle par le Collège des Jésuites, en 1800 il est transformé en Jardin Botanique et propose des cours au public. Voir : Muséum d'Histoire Naturelle de la Rochelle. Jardin des Plantes. In: Muséum d'Histoire Naturelle de la Rochelle. Site du Muséum d'Histoire Naturelle de la Rochelle. [En ligne]. [Consulté le 22 février 2013]. Disponible sur : http://www.museum-larochelle.fr/ museum/presentation/jardin-des-plantes.html

20. Voir l'article de Marie-Thérèse Vénec-Peyré, «Alcide d'Orbigny (1802-1857): sa vie et son œuvre ", Comptes Rendus Palevol, décembre 2002, volume 1, n 6, p. 313-323, p. 315.

21. Dans une lettre qu'il adresse au père d'Alcide l'Orbigny dont il est l'ami, l'entomologiste Guérin écrit ceci : «Une grande partie de la gloire scientifique doit rejaillir sur un père qui l’a poussé avec tant de succès dans la carrière des Sciences Naturelles et dont le nom, connu depuis longtemps, est pour lui la meilleure recommandation auprès des savants. " Lettre de Guérin à Charles Marie d'Orbigny, du 13 juin 1827, Médiathèque de La Rochelle, citée par Françoise Legré-Zaidline, op. cit., p. 51-52.

22. À ce sujet, voir Alcide d'Orbigny, op. cit., p. 3-5; Françoise Legré-Zaidline, op. cit., p. 9, 33, 38-39, 236 ; Marie-Thérèse Vénec-Peyré, op. cit, p. 316 ; Musée Henri Barré, Henri Barré reçoit Alcide d'Orbigny. Exposition 2006. In Ville de Thouars. Site de la ville de Thouars. [En ligne]. [Consulté le 22 février 2013]. Disponible sur : http://www.ville-thouars.fr/vah/liens/ expositions/alcideorbigny/ Alcide d'Orbigny admet lui-même avoir besoin d'une formation complémentaire pour mener à bien sa mission dans le Nouveau Monde: "L'Administration du Muséum forma le projet d'envoyer en Amérique un Naturaliste-voyageur, et me fit part de ses intentions à cet égard. Cette communication réveilla en moi l'amour des voyages, bientôt modéré, néanmoins, par le désir d'étudier encore. Je ne me trouvais pas assez instruit pour accepter une pareille mission, que j'ambitionnais de remplir le plus consciencieusement possible. Je voulais travailler quelques années de plus, afin d'acquérir, au moins en partie, les connaissances variées, nécessaires à un voyageur qui prétend servir efficacement la science et faire connaître un pays sous ses divers points de vue... " Alcide d'Orbigny, op. cit., p. 3. 
utiliseront les pratiques scientifiques, les outils de recherche et le matériel bibliographique employés dans divers pays d'Europe ${ }^{23}$. Faisant amplement appel à la population locale afin de connaître l'existence, les propriétés et l'usage de nombreux spécimens ${ }^{24}$, ils appliqueront les procédures scientifiques de dénomination des espèces et renommeront celles portant des noms indiens ou espagnols au moyen de formulations utilisées par la communauté savante, afin qu'elles soient reconnues par la Science ${ }^{25}$. La construction de l'identité nationale par le biais de la création de musées d'histoire naturelle reposera donc en grande partie sur un savoir venant d'Europe, ce qui semble contredire la volonté de créer une identité propre et indépendante mais qui permettra aussi la connaissance et reconnaissance de cette identité spécifique. Gay, d'Orbigny, Vilardebó, Isabelle, Ferraris, resteront en effet toujours liés aux communautés scientifiques de leurs pays respectifs.

23. Les conseils de méthode font d'ailleurs l'objet d'une brochure réalisée en 1824 à l'intention des scientifiques envoyés en mission par le Muséum de Paris, il s'agit d'une «Instruction pour les Voyageurs et les employés dans les colonies sur la manière de recueillir, de conserver et d'envoyer les objets d'histoire naturelle, rédigée sur l'invitation de Son Excellence le Ministre de la Marine et des colonies par l'administration du Muséum Royal d'Histoire Naturelle » Cette brochure sera rééditée jusqu'en 1860. Françoise Legré-Zaidline, op. cit., p. 39.

24. Â ce sujet, voir notamment Diego Barros Arana, op. cit., p. 276, 330-331.

25. Après les Indépendances, les naturalistes continueront d'utiliser le système de classification de Linné déjà employé pendant la période coloniale : les naturalistes espagnols y ont recours depuis 1781 . Ce système vise à maîtriser totalement la nature en intégrant tous ses éléments dans un système taxinomique préconçu. Voir à ce sujet Mauricio Nieto. Historia Natural y política: conocimientos y representaciones de la naturaleza americana. In Biblioteca virtual Luis Ángel Arango. Site de la Biblioteca virtual Luis Ángel Arango. [En ligne]. [Consulté le 22 février 2013]. Disponible sur : http://www.lablaa.org/blaavirtual/exhibiciones/historia-natural-politica/ ; Mauricio Nieto, Remedios para el imperio..., op. cit., p. 112-113, 118 ; Cécile Petit, « Les musées d'histoire naturelle et la construction des identités nationales en Amérique Latine ", Actes du Colloque L'unité dans la diversité ou la vision humboldtienne du cosmos. Identité-individualitéinterdépendance, Collège Humboldt, Paris/Evry, 17-20 juin 2009, sous presse. Comme nous pouvons le lire dans l'article de Mario Berríos et Zenobio Saldivia, cette appropriation et cette intégration ne sont pas sans poser problème : "La Historia Física y Política integra, por una parte, el conocimiento popular sobre la flora y la fauna, y por otro la descripción científica. (...) Gay utilizó las fórmulas científicas de su tiempo y de su comunidad científica parisina. Era un ilustrado y, como tal, describe, aplica técnicas y conocimientos. El hizo entrar en la universalidad a nuestro boldo, a los maitenes, al quillay, a la palma chilena y al león sin melena (puma). Frente a esto, un problema metodológico a resolver será determinar la autoría de la Historia Física y Política de Chile. Indudablemente el autor es Claudio Gay, pero él mismo dependía de otros en la búsqueda de materiales. Cuando Gay compraba a los niños de Valparaíso, a la gente del Amengual, especies raras a razón de un peso, la pregunta es quién es el descubridor y denominador de esa naturaleza. Fueron sus informantes "de a peso" los que le abrieron el mundo de la naturaleza chilena, los que lo guiaron, los que contaron anécdotas y también aquellos que no revelaron todo. En el trabajo de Gay, en su sistematización, confluyeron dos mundos; el del encuentro con las cosas en el plano de lo cotidiano, y aquel mundo europeo que por la vía de la nominación estableció legitimidad a esa realidad encontrada. " Mario Berríos, Zenobio Saldivia, "Claudio Gay y la ciencia en Chile ", Revista Creces, Chili, mai 1991. In Creces. [En ligne]. [Consulté le 22 février 2013]. Disponible sur : http://www.creces.cl/new/index.asp?imat= $>56 \& \mathrm{tc}=3 \& \mathrm{nc}=5 \& \mathrm{art}=452$ 


\section{Les MUSÉES D’hISTOIRE NATURELLE ET LE TRAVAIL RÉALISÉ PAR LES NATURALISTES : APPORTS ET DÉPENDANCES}

Les naturalistes réalisent un travail considérable et participent activement au développement de l'histoire naturelle en Amérique Latine, à la construction et à la prise en considération de l'identité nationale des pays avec lesquels ils coopèrent, grâce à l'organisation d'expéditions scientifiques, à la création de musées et à d'importantes publications. Mais comme on l'a dit, leurs recherches et la validation de leurs recherches dépendent de l'Europe. Soumises aux critères scientifiques du Vieux Continent, elles sont également tributaires des conseils, du matériel et des demandes européennes. Les naturalistes mènent leurs recherches grâce aux outils qu'ils se procurent en Europe, ils restent informés des connaissances et des idées scientifiques s'y développant et ils rendent compte de leurs travaux aux savants européens. Ils cherchent à obtenir la reconnaissance de la communauté scientifique européenne, pour eux mêmes et pour les nouvelles républiques latino-américaines, et contribuent non seulement au développement de la science dans ces pays mais aussi au renforcement du rayonnement scientifique de l'Europe.

Ainsi, Carlos Ferraris arrive en Argentine en avril 1826, en même temps que Pedro Carta Molina, son mentor. Carta Molina est invité en Argentine par Bernardino Rivadavia afin de créer et de diriger un Cabinet de Physique et d'Histoire Naturelle, mais il demande à ce que Ferraris l'accompagne, devienne conservateur du Cabinet et lui serve d'assistant dans son laboratoire de chimie $^{26}$. Par décret présidentiel, Ferraris est chargé d'aider Carta à la mise en place du Cabinet de Physique et est nommé conservateur des objets de la Salle d'Histoire Naturelle, qui se situe alors dans le Couvent de Santo Domingo, cabinet qui sera à l'origine du Muséum. Il s'y consacre tout particulièrement car, bien qu'officiellement créé en 1812 par Rivadavia, le musée ne fonctionne pas encore. Ferraris se charge du musée de 1826 à 1842, moment où il donne sa démission en raison de l'arrivée de Rosas au pouvoir. Sa démission n'étant pas acceptée, il le dirige jusqu'en 1842. Entre 1827 et 1833, les collections passent de 150 oiseaux, 180 coquillages et 800 insectes, à plus de 800 insectes, 800 animaux et 1500 minéraux, le nombre des spécimens végétaux étant

26. Dans une lettre qu'il adresse à Rivadavia, Carta Molina écrit ceci : «Otro paisano mío desearía llevar conmigo si V. M. lo consiente, es un boticario amigo desde la juventud, sujeto muy recomendable principalmente por la calidad de su corazón. Sea en el gabinete de física, sea en el laboratorio de química, necesitaré una persona que me ayude en hacer las preparaciones y las experiencias, y este amigo (que es ahora ocupado en una botica de Bruselas) es el mejor ayudante que yo pueda desear. Si no recelara de abusar de la bondad de V. M. quisiera rogarle de acordarle el paso gratis conmigo en el barco, y de nombrarle en llegando allá, conservador del gabinete de física, y mi asistente en el laboratorio de química. Este amigo cuenta de llevar consigo algunos fondos, y de establecer una botica, lo que podrá ser de alguna utilidad en el país, el que además tendrá en él un ciudadano de una virtud decidida. » Lettre de Carta Molina à Rivadavia cité par Facultad de Matemática, Astronomía y Física. Universidad Nacional de Córdoba. Ferraris, Carlos, loc. cit. 
inconnu. En tant que taxidermiste, Ferraris assure la conservation de nombreux spécimens. Et si son travail est essentiel à la création du Muséum argentin, on observe que le musée comprend aussi une collection de 720 minéraux procédant de France ${ }^{27}$. En réunissant ces collections ornithologiques, entomologiques, conchyliologiques, minéralogiques ou botaniques, le musée construit et exalte une spécificité argentine mais attire aussi l'intérêt de la communauté scientifique européenne : la valeur du musée est soumise à sa reconnaissance par les scientifiques étrangers. Plus apprécié en Europe qu'il n'excite la curiosité des Argentins, le musée semble dépendre de son regard pour pouvoir exister ${ }^{28}$. Considéré comme le fondateur du Musée d'Histoire Naturelle d'Argentine, Ferraris permettra au Musée d'être véritablement mis en place et de fonctionner. Lorsqu'il repartira en Italie, il emportera avec lui de nombreuses espèces d'animaux vivants et embaumés qu'il remettra au Musée de Sciences Naturelles de l'Université de Turin et il créera dans le Piémont un Musée argentin de zoologie et minéralogie. Après son départ, le Muséum d'Histoire Naturelle de Buenos Aires sera laissé à l'abandon pendant une bonne vingtaine d'années ${ }^{29}$.

Alcide d'Orbigny effectue son voyage scientifique en Amérique Latine de 1826 à 1833, essentiellement en Argentine et en Bolivie. Nous nous intéresserons à la première partie de son voyage, qui s'est déroulée de 1826 à 1830 en Argentine, et à sa collaboration avec le Muséum de Buenos Aires. Alcide d'Orbigny va explorer pendant plusieurs mois la province de Corrientes, l'ouest du Parana, puis la Patagonie. Il séjourne deux fois à Buenos Aires et

27. À ce sujet j’ai consulté les sites suivants : Facultad de Matemática, Astronomía y Física. Universidad Nacional de Córdoba. Ferraris, Carlos, loc. cit. ; Museo Argentino de Ciencias Naturales Bernardino Rivadavia, loc. cit. ; Facultad de Matemática, Astronomía y Física. Universidad Nacional de Córdoba. Carta Molina, Pedro loc. cit. ; ainsi que l'étude de Raúl A. Ringuelet, "Historia, estado actual y futuro de la zoología en la República Argentina ", Acta zoológica lilloana, Ministerio de Educación y Justicia, Fundación Miguel Lillo, Tucuman, Fundación Miguel Lillo, 23, 1967, p. 5-43, p. 8-9.

28. L'importance du musée dépend, hier et aujourd'hui encore, de l'intérêt que lui accordent les scientifiques étrangers. Ainsi, Pedro de Angelis écrit ceci dans les années 1830 : «De todos nuestros recientes establecimientos, el que fijará algún día la atención de los extranjeros, así como hoy excita poco la de los nacionales, es el Gabinete de Física y de Historia Natural que se aumenta silenciosamente en el Convento de Santo Domingo » : Pedro de Angelis, Crónica politica y literaria, cité par Facultad de Matemática, Astronomía y Física. Universidad Nacional de Córdoba. Ferraris, Carlos, loc. cit. La visite de scientifiques européens comme Alcide d'Orbigny, Arsène Isabelle ou Charles Darwin est constamment mentionnée. Voir par exemple Facultad de Matemática, Astronomía y Física. Universidad Nacional de Córdoba. Ferraris, Carlos, loc. cit ; Museo Argentino de Ciencias Naturales Bernardino Rivadavia, loc. cit. Même de nos jours la présence de ces naturalistes est garante de la valeur du musée. C'est ce que l'on peut constater en lisant cette phrase tirée du quotidien El Litoral: «prueba de su importancia fueron las visitas de prestigiosos viajeros como Alcides D’Orbigny, Arsenio Isabelle y otros. » in « Un museo que nació casi junto con la Patria », El Litoral, Santa Fe, 27 mai 2009. In El Litoral. Site de El Litoral. [En ligne]. [Consulté le 22 février 2013]. Disponible sur : http://www.ellitoral.com/index.php/ diarios/2009/05/27/medioambiente/MED-01.htm

29. Voir Facultad de Matemática, Astronomía y Física. Universidad Nacional de Córdoba. Ferraris, Carlos, loc. cit. et Horacio Aguilar, op. cit., p. 7. 
c'est lorsqu'il s'y rend pour la deuxième fois fin 1828 qu'il se met en contact avec Carlos Ferraris, directeur du Musée. Il participe alors à la classification des collections de mollusques du Muséum et remet au tout nouveau musée des spécimens zoologiques afin de contribuer à ses premières collections. Mais à l'origine, ce voyage n'émane pas d'une demande faite par le gouvernement argentin. Alcide d'Orbigny est en effet envoyé en mission par le Musée d'Histoire Naturelle de Paris. Le but initial est moins de contribuer au développement de la science en Argentine que d'augmenter les collections du Muséum de Paris et d'approfondir les connaissances françaises en matière d'Histoire Naturelle. Alcide d'Orbigny reçoit ainsi des instructions quant au travail à effectuer : Cuvier lui demande de lui faire parvenir en deux exemplaires les spécimens qu'il récolte, auxquels il doit joindre des notes explicatives indiquant notamment leur lieu d'origine ${ }^{30}$. D'Orbigny restera quasiment un an dans la province de Corrientes, ce qui lui permettra de recueillir de nombreux objets qu'il enverra au Muséum d'Histoire Naturelle de Paris accompagnés de leurs noms en espagnol et en guarani, de croquis et de différentes informations sur leur utilité et leur milieu naturel. En novembre 1828, il envoie plus de 50 espèces de mammifères et 250 espèces d'oiseaux au Muséum parisien. Puis en février 1830, une fois son séjour en Argentine terminé, il expédie de Valparaiso 67 oiseaux, 64 poissons, 15 reptiles, des cétacés, des mammifères et des rongeurs. Bien qu'il participe à la formation et à la classification des premières collections du Museo Argentino de Ciencias Naturales, l'essentiel de sa mission consistera à collecter, décrire, dessiner des espèces animales et végétales qu'il enverra directement au Musée d'Histoire Naturelle de Paris, où ses collections seront à nouveau étudiées. D'ailleurs, à la fin de son voyage d'exploration, il viendra présenter ses recherches à l'Académie des Sciences de Paris, où une commission de cinq personnes les examinera et jugera qu'elles permettent de compléter les collections existantes. Les recherches effectuées par d'Orbigny se font donc pour l'Académie des Sciences et le Muséum d'Histoire Naturelle auxquels il doit rendre des comptes. Elles lui ouvriront des portes et lui donneront prestige et reconnaissance. À son retour d'Amérique Latine, des naturalistes le convient à des dîners et il fait désormais partie de divers milieux scientifiques comme celui des Brongniart. En 1834, il reçoit le grand prix annuel de la Société de Géographie, créée en 1821. La Société Géologique de France, fondée en 1830 et à laquelle participent de nombreux intellectuels étrangers, l'accueille parmi ses membres. L'avis de l'Académie des Sciences lui étant particulièrement favorable, le ministre de l'Instruction Publique, Guizot, accepte de financer son projet d'édition et il pourra ainsi publier de 1835 à 1847

30. À ce sujet j'ai consulté le livre de Françoise Legré-Zaidline, op. cit., p. 9, 38-39, 52, 66, 72,75 . Voir également : « Se inaugura muestra sobre naturalista francés clave para la ciencia del siglo XIX ", Corrientes Hoy, $1^{\text {er }}$ décembre 2008, in Corrientes Hoy. Site de Corrientes Hoy. [En ligne]. [Consulté le 22 février 2013]. Disponible sur : http://www.corrienteshoy.com/vernota. asp?id_noticia=13082 ; l'étude de Marie-Thérèse Vénec-Peyré, op. cit., p. 316 et le site suivant : Facultad de Matemática, Astronomía y Física. Universidad Nacional de Córdoba. Ferraris, Carlos, loc. cit. 
ses mémoires décrivant son expédition américaine. Son Voyage en Amérique méridionale lui vaudra la reconnaissance de ses successeurs. En 1842 et 1843 il devient vice-président puis président de La Société Géologique de France. La reconnaissance dont il fait l'objet se mesure également à la création d'un genre botanique portant son nom, Orbignya, et au fait que la dénomination de 54 plantes comprenne son patronyme ${ }^{31}$. Salué pour ses apports de type ethnologique, linguistique, géologique et surtout paléontologique ${ }^{32}$, il sera néanmoins confronté à l'inimitié de certains de ses collègues scientifiques et sa candidature à l'Académie des Sciences ayant été refusée à sept reprises, il devra patienter jusqu'en 1853 pour que le Muséum d'Histoire Naturelle de Paris crée spécialement pour lui une chaire de Paléontologie et le nomme Professeur ${ }^{33}$. Il sera décoré de la Légion d'honneur ${ }^{34}$.

Quant à Claude Gay, il s'embarque pour le Chili en 1828, à la suite de sa rencontre à Paris avec Pierre Chapuis, un libéral français qui lui propose de venir enseigner la physique et l'histoire naturelle dans le tout nouvel établissement, composé de classes préparatoires et supérieures, qu'il a créé à Santiago. Cette offre l'intéresse car elle représente pour lui l'opportunité de mener des recherches zoologiques et botaniques dans un pays peu connu des Européens. Pour prendre sa décision, il demande conseil à certains de ses professeurs du Musée d'Histoire Naturelle de Paris. Ceux-ci l'y encouragent et lui donnent le titre de collecteur correspondant du Muséum. Avant d'arriver au Chili, il passe par Rio de Janeiro et Montevideo, où il commence à recueillir un certain nombre de spécimens et d'où il réalise un premier envoi de 400 plantes à destination du muséum parisien, dont beaucoup sont inconnues des scientifiques européens. Il arrive au Chili en décembre 1828. Il a peu d'élèves et le temps libre dont il dispose lui permet de se consacrer à sa passion. Dans un premier temps, il parcourt les environs de Santiago, rassemble des espèces animales et végétales qu'il classe, dessine et colore. Par le biais d'un célèbre

31. J'ai consulté les travaux de Françoise Legré-Zaidline, op. cit., p. 63-64, 75, 84, 86, $135-$ 136, 138, 141-142, 147, 151-152 et de Marie-Thérèse Vénec-Peyré, op. cit., p. 317-318.

32. Voir Françoise Legré-Zaidline, op. cit., p. 216-217 et Musée National d'Histoire Naturelle de Paris, op. cit. Jean Michel Pacaud, « Nouveautés nomenclaturales et taxinomiques introduites par Alcide d'Orbigny dans le Prodrome $(1850,1852)$ pour les espèces du Paléocène et de l'Éocène ", Geodiversitas, 29 (1), 2007, Publications Scientifiques du Muséum national d'Histoire naturelle, p. 17-85, p. 19.

33. On peut lire, sur le site du Musée National d'Histoire Naturelle de Paris : « En près de 20 ans, entre 1838 et 1857, Alcide d'Orbigny s'est présenté sept fois à l'Académie des Sciences. Sa persévérance ne fut pas récompensée puisque sa candidature fut rejetée quatre fois dans la section Zoologie et trois fois dans la section Géologie et Minéralogie. Mais c'est en 1853, quatre ans avant sa mort, qu'un décret du chef de l'État crée au Muséum la première chaire de paléontologie destinée à Alcide d'Orbigny, officialisant par là, non seulement une nouvelle discipline mais encore désignant Alcide d'Orbigny comme l'un des pères de la paléontologie. » Musée National d'Histoire Naturelle de Paris, op. cit. Voir aussi Marie-Thérèse Vénec-Peyré, op. cit., p. 321.

34. Voir Patrick Dorléans : Le retour d'Alcide d'Orbigny (1802-1857). In Association Géologique du Cher. Site de l'Association Géologique du Cher, [En ligne]. [Consulté le 22 février 2013]. Disponible sur : http://geol.cher.free.fr/ressource/orbigny.html 
pharmacien de Santiago, Claude Gay fait la connaissance du ministre Diego Portales, à qui il propose de réaliser une étude scientifique du Chili et de mettre sur pied un Cabinet d'Histoire Naturelle présentant les ressources de la nation. En 1830, le gouvernement chilien accepte son offre et l'embauche. En signant ce contrat, Claude Gay s'engage à réaliser un voyage scientifique d'une durée de trois ans et demi dans le but de répertorier de façon exhaustive les ressources naturelles du Chili, d'effectuer un cadastre pour l'État, de créer un Cabinet d'Histoire Naturelle pour y rassembler de manière organisée les principales espèces animales et végétales du pays. Cette mission lui fait traverser tout le Chili entre 1830 et 1842 et lui permet de constituer d'importantes collections animales et végétales. Claude Gay réunit 5 quadrupèdes, 213 oiseaux, 21 reptiles, 47 poissons, 2557 invertébrés et 1320 espèces végétales, 35 bois et 3 résines. Afin qu'il puisse conserver, étudier, classer et exposer les spécimens récoltés, Diego Portales demande à ce qu'un bâtiment soit construit. Ce bâtiment, inauguré fin 1839, accueillera le Musée d'Histoire Naturelle ainsi que la Bibliothèque Nationale. Toutes les pièces rassemblées par Claude Gay ne s'y trouvent cependant pas car il en remettra au Musée d'Histoire Naturelle de Paris où elles seront étudiées par ses collaborateurs. Jusqu'au début de l'année 1832, il transmet en effet les objets réunis ainsi que ses comptes rendus de recherche et de voyages à la Commission scientifique créé par le gouvernement chilien pour veiller à la bonne exécution du contrat. Ces informations sont ensuite régulièrement publiées dans le journal El Araucano. Mais en tant que correspondant du Muséum de Paris, Claude Gay doit faire part de ses recherches et remettre ses dessins et ses collections de plantes, d'animaux ou de fossiles aux institutions scientifiques françaises. Lorsqu'il revient en France en 1833, son travail est examiné par quatre membres de l'Académie des Sciences, Brongniart, Jussieu, de Blainville, Savary ${ }^{35}$, qui lui donnent de nouvelles instructions à suivre $^{36}$. À son retour en France en 1842, il présentera à nouveau ses recherches à l'Académie des Sciences et à la Société de Géographie de Paris. Il reste attaché à ces institutions, non seulement parce qu'il souhaite continuer de bénéficier de leur savoir et de leurs outils de recherche, que ce soit des livres ou du matériel,

35. À ce sujet, j'ai consulté les documents suivants : Dirección de Bibliotecas, Archivos y Museos. Memoria Chilena, loc. cit.; Biblioteca del Congreso Nacional de Chile, op. cit. Rosario Mena, loc. cit. Mario Berríos, Zenobio Saldivia, loc. cit.; Diego Barros Arana, op. cit., p. 274-279, 316-317, 331, 333-334, 347 ; Dirección de Bibliotecas, Archivos y Museos. Museo Nacional de Historia Natural de Chile, op. cit. ; Sol Serrano, op. cit., p. 113-114 ; Rafael Sagredo Baeza, " Geografía y nación. Claudio Gay y la primera representación cartográfica de Chile ", Estudios Geográficos, vol LXX, 266, enero-junio 2009, p. 231-267, p. 233-236 et du même auteur : «El arte de un naturalista ", op. cit.

36. Claude Gay s'exprime en ces termes : « De vuelta a Chile en 1833, restablecí, como era natural, mis tareas con el mayor ahinco, aunque siguiendo estrictamente un plan trazado esta vez de acuerdo con varios sabios de Paris, plan que consistia en recorrer una por una las nueve provincias de que consta la república, asentar sucesivamente en cada cual de las capitales mi observatorio, y recojer, de concierto con algunas personas intelijentes, una serie de observaciones siete y ocho veces repetidas por cada dia, y ademas renovadas, rectificadas sin cesar, durante mi permanencia en un mismo punto ». Claudio Gay, op. cit., Prólogo, p. IX. 
mais aussi parce qu'il en espère une reconnaissance, notamment par le biais de publications ${ }^{37}$. Pour remplir son contrat avec le gouvernement chilien, qui accepte de continuer de le financer bien que la durée initialement prévue soit écoulée, il rédige et publie son Historia Física y Política de Chile, œuvre en 30 volumes sur l'identité chilienne. Cet ouvrage compte 8 volumes consacrés à la botanique, 8 volumes sur la zoologie, un atlas dédiés à la botanique qui permet d'identifier et de décrire 3767 espèces végétales, alors que seules 300 environ avaient été précédemment définies. Le ministre de Instrucción Pública considère que le Chili en tant que jeune république doit disposer de ce type d'ouvrage, comme c'est le cas des pays d'Europe, pris pour modèles ${ }^{38}$. Le Chili continue de dépendre du regard européen et son objectif est avant tout d'attirer des immigrés européens ${ }^{39}$. D'ailleurs, afin de réaliser son ouvrage, Claude Gay se tourne vers des experts français. Il s'installe près du Musée d'Histoire Naturelle de Paris afin d'avoir facilement accès à sa bibliothèque et de prendre conseil auprès de ses chercheurs et il fait appel aux services de certains scientifiques spécialisés dans différents domaines de l'histoire naturelle. Il a également recours aux graveurs et aux dessinateurs parisiens pour ses illustrations et il travaille avec des imprimeurs français. Claude Gay procède aux premières études approfondies de zoologie, botanique, géologie et géographie du Chili et à ce titre il est considéré comme étant à l'origine de la science chilienne. Ses travaux, qui vont au delà des sciences naturelles, ont permis de définir

37. On peut lire dans l'étude de Diego Barros Arana : «Para ser recibido favorablemente por esos sabios, Gay tenia un medio infalible, exhibir los objetos de historia natural que habia recojido i dar a conocer sus trabajos. Fué esto lo que hizo. Comenzó por obsequiar al Museo un número considerable de minerales i fósiles, una valiosa coleccion de animales disecados, un cóndor vivo tomado en el nido en las cordilleras, i cerca de mil especies de plantas y la mitad por lo menos nueva para la ciencia europea. Del mismo modo, hizo donacion de los dibujos i pinturas en que habia reproducido un gran número dc plantas, que por su delicado tejido i sus brillantes colores, era dificil conservar en los herbarios. Obsequió tambien un rico surtido de semillas, muchas de las cuales jerminaron pronto, i pocos meses despues numerosas plantas nuevas enriquecian los jardines del Museo. Estos preciosos obsequios le abrieron todas las puertas. En los primeros meses de 1533, Gay pudo publicar en los Annales del Muséum d'histoire naturelle un resúmen analítico de sus trabajos cientificos durante todo su viaje ", Diego Barros Arana, op.cit., p. 316-317. Voir également: Biblioteca del Congreso Nacional de Chile, loc. cit. ; Rosario Mena, loc. cit. ; Mario Berríos, Zenobio Saldivia, loc. cit. ; Rafael Sagredo Baeza, "Geografía y nación... ", op. cit., p. 236 et Sol Serrano, op. cit., p. 114 .

38. À ce sujet j'ai consulté : Biblioteca del Congreso Nacional de Chile, loc. cit. ; Rosario Mena, loc. cit. ; Rafael Sagredo Baeza, «El arte de un naturalista », loc. cit. ; Diego Barros Arana, op. cit., p. 241, 428.

39. Cet objectif figure sur le contrat conclu entre Claude Gay et le gouvernement chilien : "Art. 1. ${ }^{\circ}$ Don Claudio Gay se obliga a hacer un viaje científico por todo el territorio de la república, en el término de tres ańos i medio, con el objeto de estudiar la historia natural de Chile, su jeografía, jeolojía, estadística i cuanto contribuya a dar a conocer las producciones naturales del pais, su industria, comercio i adrninistracion (...) Art. $3^{\circ}$. Siendo uno de los objetos del gobierno al confiar esta importante comision a don Claudio Gay, dar a conocer las riquezas del territorio de la república, para estimular la industria de sus habitantes i atraer la de los estranjeros, don Claudio Gay se obliga a publicar su obra tres años despues de concluida su comision. »Contrat du 14 septembre 1830, cité par Diego Barros Arana, op. cit., p. 277-278. 
les spécificités de la nature chilienne. Mais cette oeuvre est une fois encore élaborée par un Européen. Elle permettra à son auteur d'être mondialement reconnu comme scientifique et comme naturaliste. Claude Gay obtiendra les félicitations de Humboldt et recevra en 1845 la médaille d'or de la Société de Géographie de Paris. Néanmoins, en 1854, sa première demande pour devenir membre officiel de l'Académie des Sciences est refusée. Elle sera acceptée en 1856. Il devient également membre de l'Institut de France et reçoit la Légion d'honneur du gouvernement français ${ }^{40}$.

Teodoro Vilardebó, revient en Uruguay, son pays d'origine, en 1833. Docteur en médecine, il travaille comme médecin à Montevideo de 1833 à 1844, est nommé membre de la Junta de Higiene, mais a pour ambition de réaliser une histoire politique et naturelle de l'Uruguay. Ses activités de naturaliste paléontologiste se font parallèlement à son activité professionnelle officielle. A partir de 1837, il se consacre davantage à l'histoire naturelle de l'Uruguay, et en particulier à l'étude des fossiles, en raison de sa nomination à la Comisión de Biblioteca y Museo ${ }^{41}$. Cette commission est créée par décret en septembre 1837 afin de mettre en place un musée d'histoire naturelle et une bibliothèque. Elle est chargée de rassembler et d'étudier des pièces végétales, minérales et animales afin d'entreprendre la constitution d'un cabinet d'histoire naturelle. Ces travaux et le matériel nécessaire seront financés par le gouvernement qui mettra à disposition un local pour exposer les objets rassemblés ${ }^{42}$. Une découverte paléontologique réalisée fin 1837 sera décisive.

40. J'ai consulté : Biblioteca del Congreso Nacional de Chile, loc. cit.; Rosario Mena, loc. cit.; Mario Berríos, Zenobio Saldivia, loc. cit. ; Diego Barros Arana, op. cit., p. 418-419, 424-425, p. $441-442$.

41. Voir à ce sujet : Alberto F. Onna., op. cit., p. 60, 64 ; Juan María Gutiérrez, op. cit., p. 279280 ; Alvaro Kröger, loc. cit. ; María L. Coolighan Sanguinetti, op. cit., p. 33-35.

42. J'ai consulté : Pedro Mascaró y Sosa, «Apuntes para una historia de la biblioteca nacional de montevideo ", Anales del Ateneo del Uruguay, Año 1, tomo 2, n 1, mai 1882, p. 161-184, p. 173. Voici le décret dont il est question : « No pudiendo el Gobierno retardar por mas tiempo el establecimiento de una Biblioteca pública, á cuya medida se habian opuesto hasta ahora, graves y poderosos motivos, y considerando en medio de las multiplicadas atenciones que le rodean, que el modo mas adecuado para la ereccion de esta institucion, y la de un Museo de historia natural, de cuya importancia se halla tambien penetrado, es el de apelar á la generosidad y patriotismo de los Ciudadanos, que no pueden menos que corresponder á las miras y esperanzas de la autoridad, desde que de otra manera se vería á su pesar, en la necesidad de postergar para mas adelante esta importante medida, ha venido en acordar y decreta:

Art. $1 .^{\circ}$ Nombrase una Comision, que se denominará de Biblioteca y Museo, compuesta de los ciudadanos. D. Ramon Masini, D. Bernardo Berro, D. Manuel Erasquin, D. Cristoval Salvañach, y Dr. D. Teodoro Vilardebó.

2. ${ }^{\circ} \mathrm{La}$ Comision se encargará de colectar de los particulares, todas las obras que voluntariamente quieran donar, para la ereccion de la Biblioteca pública, y de inquirir y recavar á la vez de los mismos, los objetos de mineralogía, botanica y zoología de que quieran desprenderse, para poder hechar las bases de un gavinete de historia natural.

3. ${ }^{\circ}$ El Gobierno despues de conocido el resultado de los esfuerzos patrioticos de la Comision, destinará inmediatamente un local adecuado para ambos establecimientos, y designará con anuencia previa del cuerpo Legislativo, las sumas que considere necesarias para sostenerlos y 
Le squelette d'un animal étrange et non identifié est trouvé en décembre 1837 dans le département de Canelones situé au nord de Montevideo. Vilardebó est envoyé sur place avec un autre membre de la commission, Berro, pour étudier ce squelette. Il s'agit d'un fossile de glyptodonte, ancêtre du tatou. Ils rédigent un compte rendu comportant une description détaillée de ce spécimen qu'ils nomment Dasypus antiquus, suivant ainsi les normes de dénominations de Linné utilisées en Europe. En l'absence de revues scientifiques spécialisées, leur rapport sera diffusé dans la presse généraliste chilienne ${ }^{43}$. Cette étude sera capitale et lui permettra d'être nommé en 1839 président de la Commission du Musée d'Histoire Naturelle ${ }^{44}$. En 1843, il fondera l'Institut Historique et Géographique puis, en 1845, il sera nommé membre de l'Institut Historique et Géographique de Rio de Janeiro. Vilardebó cherchera à faire connaître cette étude et ses travaux ultérieurs aux communautés scientifiques européennes. Passant vingt-quatre ans de sa vie en Europe et quinze ans de sa vie d'adulte en Uruguay, ses contacts lui permettent d'être en relation avec les milieux scientifiques européens et de diffuser le résultat de ses recherches. Par le biais d'Alcide d'Orbigny, l'Académie des Sciences de Paris est, par exemple, informée de la découverte de nouveaux fossiles. La légitimation de ses travaux passe par la reconnaissance scientifique française ${ }^{45}$. Lors d'un séjour qu'il effectuera en Europe entre 1847 et 1853 , il vendra sa collection de fossiles uruguayens au Musée d'Histoire Naturelle de Paris ${ }^{46}$.

Arsène Isabelle arrive en Uruguay en 1830, pour son propre compte et à ses frais. Son but est de se lancer dans la production et le commerce de savon, de chandelles, et dans l'exportation de suif, projet qui se solde par un échec. Dans le même temps, il souhaite se consacrer à sa passion pour le voyage et pour l'histoire naturelle. De 1830 à 1834, il parcourt l'Argentine, le Brésil et l'Uruguay, dans l'objectif de recueillir l'ensemble des spécimens végétaux et zoologiques des lieux qu'il traverse. Pour ce faire, il s'équipe du matériel nécessaire pour chasser, naturaliser les animaux et conserver la flore ramassée. Il emporte avec lui des livres d'histoire naturelle et bénéficie de l'aide de

enriquezcer el caudal de libros y objetos de historia natural, á fin de darle todo el aumento de que es susceptible.

4. ${ }^{\circ}$ La expresada Comision, formará un presupuesto de los primeros gastos que exija la colocacion de estos objetos, y mas adelante otro de aquellos que anualmente demande de su conservacion é incremento, indicando al mismo tiempo la clase y número de empleados que sean indispensables para dirigir, y servir dichas instituciones. " Ministerio de Gobierno, "Decreto que nombra una Comisión de Biblioteca y Museo público ", Registro Nacional, tomo 2, n 3, Montevideo, setiembre de 1837, p. 181-182.

43. Voir le travail de Alberto F. Onna, op. cit., p. 65.

44. J'ai consulté l'étude de Pedro Mascaró y Sosa, op. cit. p. 173.

45. Voir Alberto F. Onna, op. cit., p. 61, 64-65, 67.

46. On peut lire cette information dans les documents suivants: María L. Coolighan Sanguinetti, op. cit., p. 37 et Museo Nacional de Historia Natural y Antropología de Uruguay. Teodoro Miguel Vilardebó. In: Museo Nacional de Historia Natural y Antropología de Uruguay. Site du Museo Nacional de Historia Natural y Antropología de Uruguay. [En ligne]. [Consulté le 22 février 2013]. Disponible sur : http://www.mec.gub.uy/munhina/biograf14.htm\#Teodoro M. Vilardebó 
naturalistes et de scientifiques comme Bonpland, Fabrizio Mossoti ou Carlos Ferraris ${ }^{47}$. Rentré en France, il écrit son livre Voyage à Buénos Ayres et à Porto Alegre, par la Banda Oriental (1835). Y sont abondamment décrites les collectes de la flore et la faune ${ }^{48}$. Sa préoccupation majeure est néanmoins de type commercial : il souhaite avant tout signaler les bénéfices que l'industrie et le commerce français et havrais pourraient tirer des richesses de ces territoires ${ }^{49}$. Revenant en Uruguay en 1837 en tant que consul français, il participe à titre personnel à la première expédition scientifique du Museo Nacional de Historia Natural de Uruguay en 1837 et publie avec Teodoro Vilardebó l'un des premiers textes sur le glyptodonte ${ }^{50}$. Entre 1837 et 1839, il recueille des espèces végétales qu'il remettra au Muséum d'Histoire Naturelle de Montevideo et qui font partie des collections les plus anciennes du musée ${ }^{51}$. Il donnera une centaine de spécimens uruguayens au musée botanique de Benjamin Delessert, institution européenne majeure disposant de collections de plantes et d'une bibliothèque auxquelles tous les botanistes de l'époque auront recours ${ }^{52}$.

47. Voir à ce sujet Arsène Isabelle, op. cit., p. 11-12, 14-17, 29, 31-32 et Horacio Aguilar, op. cit., p. 6.

48. Voir Horacio Aguilar, op. cit., p. 7.

49. Arsène Isabelle : « Occupé depuis mon retour à mettre en ordre mes nombreuses notes, j'ai toujours eu en vue d'en faire profiter mes compatriotes et principalement le Commerce du Havre, qui par son heureuse position et son extension, parait avoir un intérêt plus direct à bien connaître des lieux destinés peut-être à augmenter beaucoup sa prospérité. "Arsène Isabelle, op. cit., p. 19. Et plus loin : «Je fais ressortir, autant que mes trop faibles lumières me le permettent, les avantages que notre industrie manufacturière pourrait retirer d'un système commercial bien entendu, convenablement approprié aux goûts et aux besoins de ces peuples pasteurs, agriculteurs, artisans et fainéans ", ibid., p. 25-26. Dans la dernière partie de son livre intitulée "Considérations sur l'état du Commerce Français à l'extérieur, et principalement au Brésil et au Rio-de-la-Plata ", Arsène Isabelle exprime son souhait d'améliorer le commerce français et souligne l'utilité du commerce extérieur et des exportations, sources importantes de capitaux. Ibid., p. 540-541.

50. Voir Horacio Aguilar, op. cit., p. 6 et Alberto F. Onna, op. cit., p. 65.

51. À ce sujet, j'ai consulté les pages suivantes : Museo Nacional de Historia Natural y Antropología de Uruguay. Botánica. Site internet du Museo Nacional de Historia Natural y Antropología de Uruguay. [En ligne]. [Consulté le 22 février 2013]. Disponible sur : http:// www.mec.gub.uy/munhina/bot.htm et Museo Nacional de Historia Natural y Antropología de Uruguay. Arsène Isabelle. Site internet du Museo Nacional de Historia Natural y Antropología de Uruguay. [En ligne]. [Consulté le 22 février 2013]. Disponible sur: http://www.mec.gub.uy/ munhina/biograf9.htm\#Arsène Isabelle.

52. Dans son ouvrage sur le musée botanique de Benjamin Delessert, Antoine Lasègue mentionne la présence de spécimens rassemblés par Arsène Isabelle : "Les plantes de M. Isabelle ont été récoltées pendant le cours de son voyage. Celles qui font partie maintenant de l'herbier de M. Delessert s'élèvent à environ 100 espèces de Montevideo et 250 de la province de Rio-Grande-do-Sul. Les lichens et les mousses ont été principalement recueillis aux environs de Porto-Allegre et de San-Leopoldo. Les fougères appartiennent à toute la province, depuis l'Uruguay jusqu'à la capitale ". Antoine Lasègue, Musée botanique de M. Benjamin Delessert, Paris : Librairie de Fortin, 1845, 588 p., p. 264. L'importance du musée botanique de Benjamin Delessert est soulignée sur le site de la Bibliothèque de l'Institut de France : « Le musée botanique de Benjamin Delessert fut, dans la première moitié du XIX ${ }^{e}$ siècle, une institution centrale dans le monde savant. Lieu d'érudition qui rassemblait de nombreux ouvrages anciens, mais 


\section{Conclusion}

Carlos Ferraris, Alcide d'Orbigny, Claude Gay, Teodoro Vilardebó et Arsène Isabelle jouent un rôle primordial dans le développement de l'histoire naturelle en Amérique Latine. Ces naturalistes participent à la création, à la direction, à l'élaboration de collections du Museo Argentino de Ciencias Naturales Bernardino Rivadavia, du Museo Nacional de Historia Natural du Chili et du Museo Nacional de Historia Natural y Antropología d'Uruguay. Étant donné que l'histoire naturelle, les musées, et notamment les musées d'histoire naturelle, ont pour but de favoriser la connaissance d'un pays et des spécificités naturelles qui contribuent à son identité nationale, ces naturalistes participent à la construction identitaire de l'Argentine, du Chili et de l'Uruguay. Cependant, en reposant sur les travaux de scientifiques européens ou formés en Europe, la construction identitaire menée par les gouvernements argentin, chilien et uruguayen ne parvient pas à se démarquer totalement de l'Europe et implique une certaine dépendance scientifique, économique et culturelle, qui est néanmoins perçue comme le passage nécessaire à la reconnaissance de leurs pays.

L'influence des naturalistes européens en Argentine, au Chili et en Uruguay se prolongera au-delà de la période de fondation des musées d'histoire naturelle. Tout au long du XIX ${ }^{e}$ siècle, dans les décennies qui suivront leur création, ces musées continueront de s'appuyer sur l'expérience de naturalistes européens. Ainsi, le Museo Argentino de Ciencias Naturales Bernardino Rivadavia aura notamment pour directeurs Antonio et Demetrio Demarchi, nés en Suisse, Herman Burmeister et Carlos Berg, originaires d'Allemagne, et bénéficiera de la collaboration de Fernand Lahille et Auguste Bravard, tous deux français. Le Museo Nacional de Historia Natural y Antropología d'Uruguay aura entre autres à sa tête Carlos Berg, précédemment cité, José Arechavaleta, naturaliste de nationalité espagnole, et aura pour collaborateurs Cornelius Osten, Ernesto Gibert, Luis Panizza et Carlos Spegazzini, respectivement allemand, français et italiens. Le Museo Nacional de Historia Natural du Chili sera quant à lui dirigé par Philibert Germain, de nationalité française, ainsi que par Rudolf et Federico Philippi, allemands.

\footnotetext{
surtout lieu où la science se faisait : les plus grands noms de la botanique s'y retrouvaient alors pour consulter le riche fonds de monographies, flores nationales et régionales, périodiques et mémoires d'académies. La bibliothèque était complétée par un herbier qui se trouve aujourd'hui au Conservatoire botanique de Genève. La collection fondée par Benjamin Delessert (17731847), banquier et mécène, ami de Alexander von Humboldt, fut poursuivie par son frère François jusqu'en 1869. À cette date, la bibliothèque fut léguée à l'Académie des sciences. " Bibliothèque de l'Institut de France. Bibliothèque botanique Benjamin Dellessert. Site de la Bibliothèque de l'Institut de France. [En ligne]. [Consulté le 22 février 2013]. Disponible sur : http://www.bibliotheque-institutdefrance.fr/fonds/fonds.html
} 